\title{
DEDICATED COSMIC RAY MEASUREMENTS FOR SPACE WEATHER AND EDUCATIONAL PURPOSES
}

\author{
F.JANSEN*, D.STIEFS, T. BRANDT, P. WINKLER \\ DLR Institute of Space Systems, 28359 Bremen, Robert-Hooke-Str.7, Germany \\ *E-mail: frank.jansen@dlr.de \\ C. TIMMERMANNS
}

Experimental High Energy Physics, Mailbox 79, P.O. Box 9010

6500 GL Nijmegen Nikhef, Radboud University, The Netherlands

S. POSPISIL

Institute of Experimental and Applied Physics, CTU Prague, Horská 3a/22, Praha 2, 12800, Czech Republic

\section{K. KUDELA}

Institute of Experimental Physics, Slovak Academy of Sciences, Watsonova 47, 04001 Košice, Slovak Republic

\begin{abstract}
This paper describes the Global Muon Detector Network (GMDN) data applied to Tohuku earthquake and Fukushima nuclear power plant failure. In addition the DLR_School_Lab is introduced by the display of Coronal Mass Ejections due to GMDN data and by means of the cosmic ray muon shower real time visualization on a planetarium dome. A Timepix hodoscope space based satellite is sketched.
\end{abstract}

\section{Introduction: GMDN - Global Muon Detector Network, Tohuku Earthquake and Fukushima Dai-ichi Nuclear Power Plant Failure}

Just before Tohuku earthquake in March 2011, respectively Fukushima Dai-ichi nuclear power plant failure T. Ebisuzaki et al. [1] published, that three most disastrous earthquakes $(1923,1964,1995)$ in the 20th century in Japan occurred in cosmic ray maximum conditions (respectively solar minimum period). In contrast to the established relationship between explosive volcanic eruptions in Japan triggered by cosmic ray muons a physical mechanism for triggering earthquakes by muons is less understood. However first proof of usability of cosmic ray neutron monitor for earthquake detection, were given by a $2 \%$ neutron intensity increase exact with the begin of the Tien Shan earthquake on 
25 December 2006 (V.P. Antonova et al. [2]). The measured neutron intensity increase originated by radioactive decay of radon isotopes just when the earthquake started.

In the case of the Tohuku earthquake, the Nagoya Scintillator Telescope (NST) of the GMDN gave the first proof of cosmic ray muon count increase originated by an earthquake: the physics \& mathematics behind the GMDN, the belonging cosmic ray muon measurements and calibration of the five ground based muon telescopes in Australia, Brazil, Germany, Kuwait and Japan were already described in detail in F. Jansen et al [3]. In Figure 1 is presented the NST data of all 17 arrival directions (summarized in vertical, north, south, east and west observational counts per hour) between 11 and 16 March 2011. Figure 2 shows hints of about $1 \%$ cosmic ray muon anisotropy during the earthquake and the first week of the Fukushima Daiichi nuclear power plant failure. This cosmic ray muon anisotropy was not induced by a coronal mass ejection (CME), which arrived on Earth one day before earthquake (10 March 2011 05:45 UT, solar wind increase from about $300 \mathrm{~km} / \mathrm{s}$ to $340 \mathrm{~km} / \mathrm{s}$ only) and disappeared in the data latest 12 March $2011(\mathrm{Kp}=2)$. Figure 3 compares Nagoya (NST) with Hobart (HST) and Sao Martinho (SMST) scintillator telescope muon counts. Both comparisons support the higher NST counts by about $1 \%$ higher only at Nagoya.

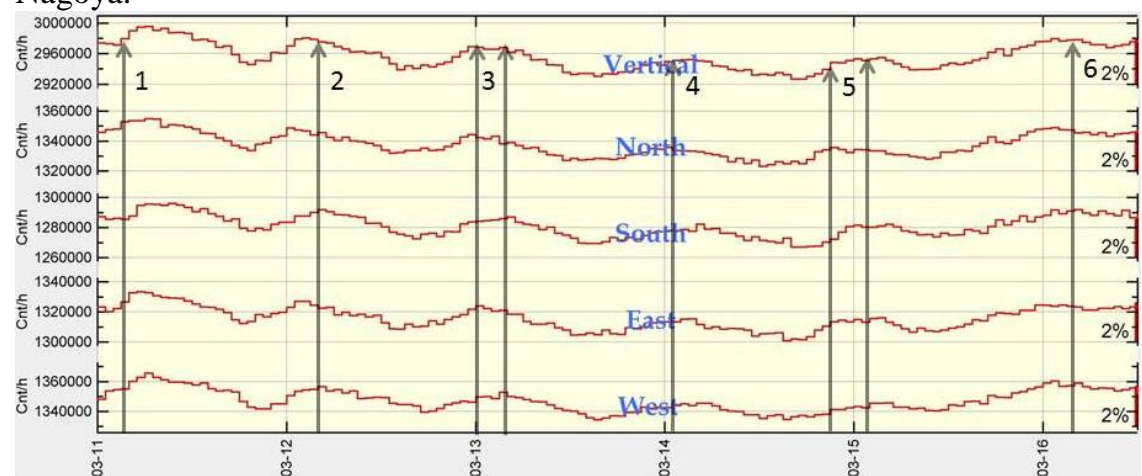

Figure 1 The NST hourly cosmic ray muon intensity measured 11 March to 16 March 2011 about $500 \mathrm{~km}$ southward from Fukushima Dai-ichi nuclear power plant during the M9 Tohuku earthquake and the explosion and ventings at Fukushima Dai-ichi nuclear power plan (vertical lines: 1 Earthquake 05:46 am UT; 2 explosion Unit1 06:36 am UT, venting Unit1 10:25 am UT, detection ${ }^{137} \mathrm{Cs}$ and ${ }^{131} \mathrm{I}$; 3 venting Unit3 and 2 00:20 am UT and 04:50 am UT; 4 H explosion Unit3 02:01 am UT; 5 explosion Unit2 9:10 pm UT 30...400mSv/h, explosion and fire Unit4 9pm to 2am; 6 venting Unit2 and 3 about 03:00 am UT. 


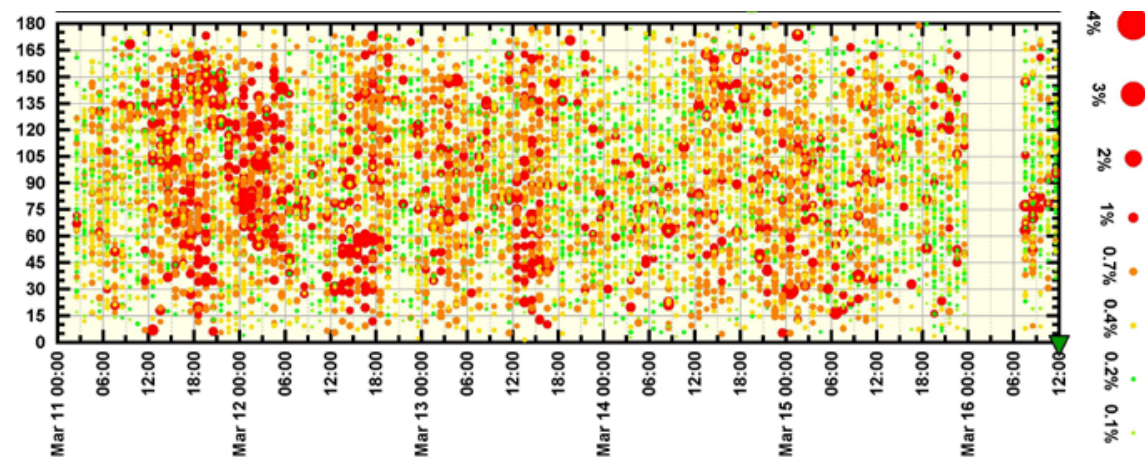

Figure 2 The ordinate is the pitch angle distribution $\left(45^{\circ}\right.$ Parker spiral field at Earth orbit, $0^{\circ}$ Earth motion direction in interplanetary space around the Sun). Enhanced anisotropies correlates with the time of the earthquake, explosions and ventings (see vertical lines in Figure 1). The enhancements are only caused by the NST data - not measured in the four other GMDN telescopes (see Figure 3).

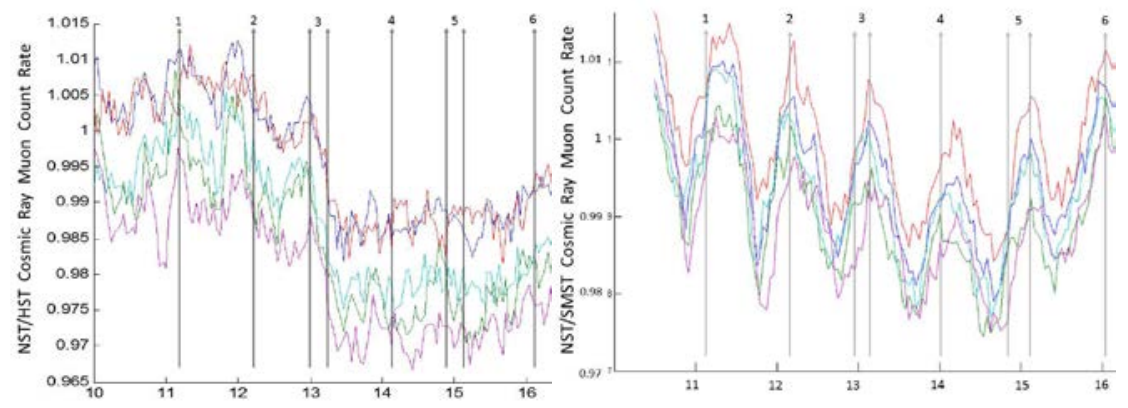

Figure 3 Left: normalized Nagoya to Hobart muon telescope data and right are displayed the normalized Nagoya to Sao Martinho muon telescope data. The 1\% enhancement of NST muon counts are visible (until about the end of 12 March 2011) in all muon incoming directions (from top to down curves are all incoming muon direction: from vertical, north, south, east and west) . The daily cosmic ray variation is not the reason for the enhancement and NST was operating normally and had no malfunctions during these days. Vertical lines like in Figure 1.

Jansen et al. [4] studied additional muon production in two processes: for the earthquake via ${ }^{222} \operatorname{Rn}(\alpha, n){ }^{218}$ Po $\left({ }^{222} \mathrm{Rn}\right.$ was observed via TEC anomalies by $\mathrm{D}$. Ouzounov et al. [5]). The free neutrons decay into additional electrons, which collided with secondary cosmic ray positrons and produces via $\mathrm{Z}^{0}$ additional $\mu^{-}$ $\mu^{+}$. During the power plant failures ${ }^{239} \mathrm{Pu} \rightarrow{ }^{235} \mathrm{U} \rightarrow{ }^{131}$ I respectively and ${ }^{239} \mathrm{Pu}$ $\rightarrow{ }^{235} \mathrm{U} \rightarrow{ }^{236} \mathrm{U} \rightarrow{ }^{137} \mathrm{Cs}$. The $\beta^{-}$decays of ${ }^{131} \mathrm{I}$ and ${ }^{137} \mathrm{Cs}$ produced additional electrons, which produced via secondary cosmic ray positrons and $\mathrm{Z}^{0}$ additional $\mu^{-} \mu^{+}$. Due to very low decay cross section $Z^{0} \rightarrow \mu^{-} \mu^{+} \sim 0.92$ nb both processes results into magnitudes to low additional muon production. Therefore, the time correspondence of the comic ray variations observed at Nagoya muon telescope with the Tohuku earthquake respectively Fukushima Dai-ichi nuclear power 
plant failure requires further investigations before the causal conclusion can be deduced.

\section{DLR_School_Lab Bremen, GMDN and Cosmic Ray Timepix Hodoscope}

In figure 4 is visualized the extended - by about $150^{\circ}$ - cosmic ray muon sky above Nagoya on 11 March 2011. Those images and videos are produced worldwide for the first time in the planetarium dome of the DLR_School_Lab in Bremen. Those images and videos are constructed and displayed by interpolation of GMDN anisotropy data points in combination of tailored visualization software. The angular resolution of all GMDN ground based telescopes is in the order of several degrees. Therefore in the future a high resolution Timepix based cosmic ray hodoscope is foreseen to be mounted on a space weather spacecraft.
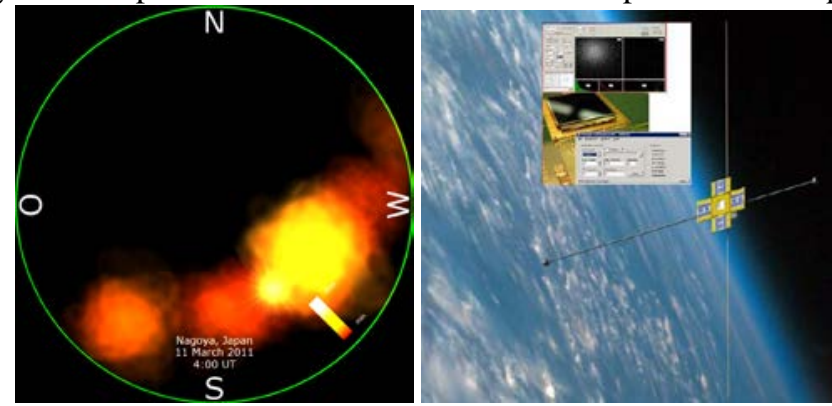

Figure 4 Left: image of the cosmic ray muon sky from all five GMDN telescopes above Nagoya about $1 \mathrm{~h} 46 \mathrm{~min}$ before Tohuku earthquake in Japan (the horizon is green). Right: spacecraft designed at DLR Institute of Space Systems in Bremen for real time CME visualization by a Timepix cosmic ray hodoscope.

\section{DLR_School_Lab Bremen and Real Time Cosmic Ray Visualization}

For space weather educational purposes the DLR_School_Lab in Bremen is equipped with a small cosmic ray muon telescope, which consists of a coincidence system with twelve plastic scintillator detectors and photomultiplier tubes. This DLR_School_Lab muon telescope visualize in real time on the planetarium dome cosmic ray muon shower for the first time worldwide. 


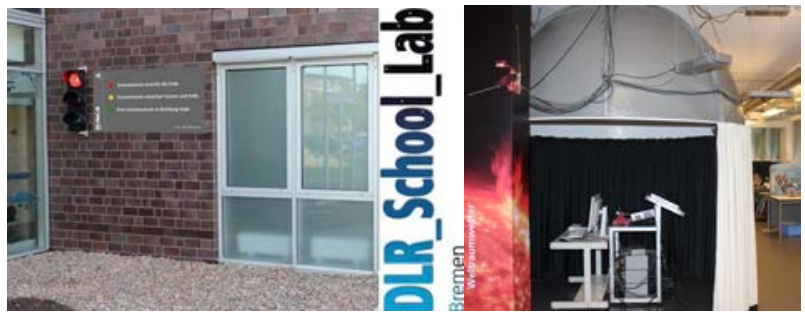

Figure 5 Left image: at the entrance wall of the DLR Institute of Space Systems in Bremen a space weather traffic signal automatically visualize by means of GMDN anisotropy data the CME location between Sun and Earth. Right image: planetarium dome with fish eye lenses, laser beamer and PC for real time visualization of cosmic ray shower by means of the two layers of plastic scintillator detectors and photomultiplier tubes (in the upper right part of the image).

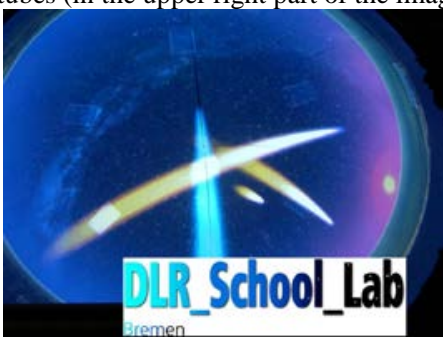

Figure 6 Inside the DLR_School_Lab Bremen planetarium dome. Examples of four different colored (as a function of the muon count rate) cosmic ray spurs due to a cosmic ray shower are visible. In addition artificial stars, the Milky Way, the Sun as well as contours of the telescope detector boxes are displayed for educational understanding.

\section{Summary}

Different cosmic ray muon measurements showed their powerful applications for space weather, educational purposes and for earthquake research as well.

\section{References}

1. T. Ebisuzaki, H. Miyahara, R. Katoaka, T. Sato, Y. Ishimine, Gondwana Research 19, 1054 (2011).

2. V.P. Antonova, N.N. Volodichev, S.V. Kryukov, A.P. Chubenkov, A.L. Shchepetov, Geomagnetism and Aeronomy 49, 761 (2009).

3. F. Jansen and J. Behrens, Cosmic Rays for Particle and Astroparticle Physics, Proc. of the $12^{\text {th }}$ ICATPP Conference, Eds: S. Giani, C. Leroy, P.G. Rancoita, 378, (2011).

4. F. Jansen, S. Pospisil, A. Weiß ,V. Maiwald, P. Beltrami, K. Munakata, Behrens, http://www.swpc.noaa.gov/sww/SWW_2012_Presentations/ SWPC workshop Boulder (2012).

5. D. Ouzounov, S. Pulinets, A. Romanov, A. Romanov, K. Tsybulya, D. Davidenko, M. Kafatos, P. Taylor, arXiv:1105.2481v1, EGU Vienna (2011). 